\title{
Helical Propulsion in a Viscous Heterogeneous Medium
}

\author{
Anke Klingner*, Dalia Mahdy ${ }^{\dagger}$, Mostafa Hanafi ${ }^{\dagger}$, \\ ${\text { Barbara } \text { Adel }^{\dagger} \text {, Sarthak Misra }}^{\ddagger \S}$ and Islam S. M. Khalil ${ }^{\ddagger}$
}

\begin{abstract}
We characterize the propulsion of externallyactuated helical robots inside a viscous heterogenous medium. The method of regularized Stokeslets is implemented in threedimensional space for computing the Stokes flow around a helical robot and immersed obstacles (spherical microparticles) in the medium. The helical robot is actuated using a permanent magnet-based robotic system with two synchronized rotating dipole fields. Our simulations and experimental results demonstrate propulsion enhancement with the concentration of the immersed obstacles in the viscous medium regardless of the actuation frequency. Numerical results show that the swimming speed is increased approximately by a factor of 2 for a $5 \%$ increase in the concentration of immersed obstacles with diameter of $30 \mu \mathrm{m}$, at actuation frequency of $1 \mathrm{~Hz}$. At this actuation frequency, our experimental results show that the swimming speed is increased by a factor of 1.4. At relatively high actuation frequency $(8 \mathrm{~Hz})$, simulation and experimental results also show increase in the swimming speed by factors of 1.4 and 1.3 , respectively.
\end{abstract}

\section{INTRODUCTION}

The ability of artificial microrobots to swim controllably in complex environments is likely to be an important advancement toward their translation into in vivo applications. Several concepts to realize untethered propulsion on a small size scale have been pursued by different research groups [1][13], where viscous drag dominates over the inertial forces. However, propulsion of these untethered devices is significantly affected by in vivo conditions such as the time-varying fluid drag [14], heterogeneity of the background fluid [15], varying vessel diameter, and channel wall effects [16]. These conditions place strong challenges on the design and development of microrobots. In addition, these conditions vary across the human body and necessitate a specific microrobot design based on the size and material of the environment. Therefore, it is essential to understand the influence of the in vivo conditions on the propulsion of the microrobots through theoretical and experimental work.

Nelson et al. have derived an empirical model to relate screw geometry and soft-tissue properties based on experimental measurements [17]. Ullrich et al. have also investigated the swimming characteristics of helical microrobots in fibrous environment, and showed that the helical propulsion is enhanced by the presence of collagen fibers in a gelatine

The authors are affiliated with the *Physics Department and ${ }^{\dagger}$ Mechatronics Department, The German University in Cairo, Cairo, Egypt.

${ }^{\ddagger}$ The authors are affiliated with the Department of Biomechanical Engineering, University of Twente, 7522 NB Enschede, The Netherlands.

$\S$ The author is affiliated with the Department of Biomedical Engineering, University of Groningen and University Medical Centre Groningen, 9713 AV Groningen, The Netherlands.
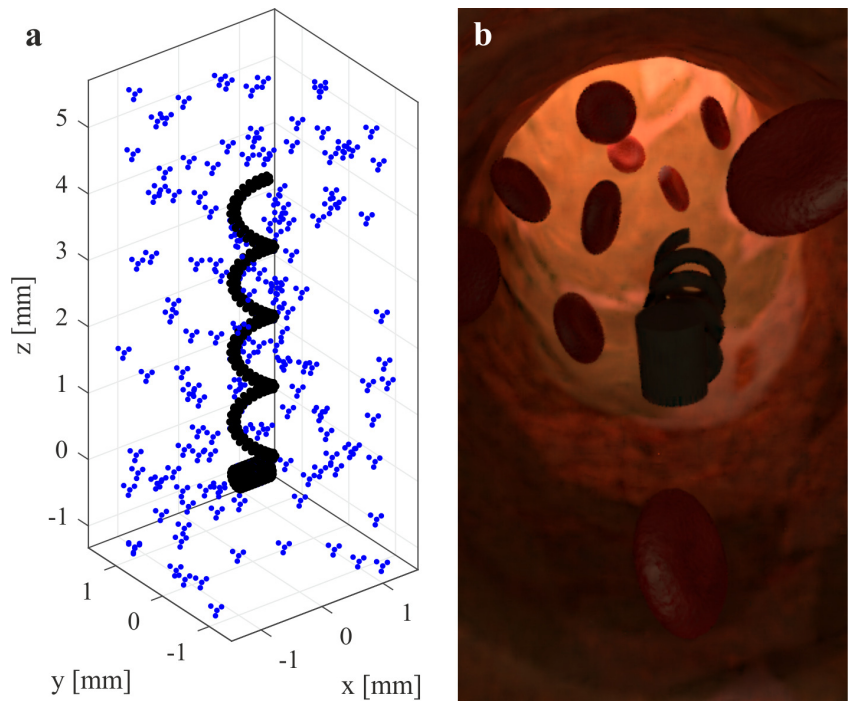

Fig. 1. A numerical model of the helical robot is developed based on the method of regularized Stokeslests. This model predicts the influence of the concentration of immersed obstacles within a viscous medium on the propulsion of the helical robot. (a) The helical robot and the obstacles are represented using the black dots and blue tetrahedrons, respectively. The tetrahedrons are used to model the spherical obstacles within the medium. (b) Helical microrobots have potential biomedical applications owing to their ability to physically access small spaces in a non-invasive manner.

with medium concentration due to movements similar to corkscrew motion without slippage [18]. Motile bacteria have also shown increased swimming speed with the increased viscosity due to their interaction with the fibrous network of the background fluid [19]. This network enables bacteria to push themselves off the surrounding obstacles and change the pitch of the helical motion. Leshansky has also presented a theoretical framework to predict the behaviour of rigid bodies in heterogenous viscous environment based on solutions of averaged equations of viscous flow through a random spare array of obstacles [15]. However, the mentioned theoretical work has not been compared to experimental results. Similarly to motile bacteria, soft microrobots have also shown propulsion enhancement in low Reynolds numbers viscous heterogeneous medium. This propulsion enhancement is attributed to the local pressure field created at the distal tip of the beating flexible tail of the microrobots at relatively low actuation frequency owing to the increased amplitude along the length of the tail. Soft microrobots create relatively high flow-field in close proximity to the distal end of the tail as opposed to the head where the propagating wave 
originates [20]. In this work, we present a numerical model based on the method of regularized Stokeslets [21] for helical propulsion inside a viscous heterogenous medium. This model allows us to investigate the influence of the concentration of immersed obstacles (Fig. 1(a)) within the medium on the swimming speed of helical robots actuated using rotating dipole fields. The theoretical predictions of this numerical model are validated by experimental results. These experiments are conducted on a permanent magnetbased robotic system with two synchronized rotating dipole fields to actuate helical robots inside catheter segments.

The remainder of this paper is organized as follows: Section II provides the numerical model of the helical robot and description of the regularized Stokeslets method. Simulation results are also provided in Section II to study the influence of the obstacles concentration and the actuation frequency on the helical propulsion. Section III includes descriptions pertaining to the permanent magnet-based robotic system and the helical robot. It also provides our experimental results and discussions on the experimental validation of the theoretical predictions. Finally, Section IV concludes and provides directions for future work.

\section{HelicAl Propulsion in A Viscous Heterogeneous Medium}

Helical robots are allowed to swim inside catheter segments under the influence of rotating magnetic fields. The heterogeneity of the background fluids is one of the features that will be encountered in vivo (Fig. 1(b)).

\section{A. Stokeslets flow-fields}

The helical robot consists of a cylindrical head of length $2 r_{\mathrm{c}}$ and diameter $L_{\mathrm{c}}$. The head is attached to a helix with length $L$ and diameter $2 H$, as shown in Fig. 2. The axial magnetization of the head of the helical robot $\mathbf{m}$ is orthogonal to the helix axis, and allows for rotation with an angular frequency, $\omega=2 \pi f$, with the external magnetic field $\mathbf{B}$. This field exerts a magnetic torque $(\mathbf{m} \times \mathbf{B})$ on the magnetic dipole of the helical robot. The robot is contained inside a mixture of viscous medium and spherical microparticles with an average diameter of $2 r_{\mathrm{p}}$ and volume concentration $\varphi=V_{\mathrm{p}} / V$, where $V_{\mathrm{p}}=\frac{4}{3} \pi r_{\mathrm{p}}^{3} N_{\mathrm{p}}$ is the total volume of the microparticles in the mixture, $V$ and $N_{\mathrm{p}}$ are the volume of the viscous medium and the number of microparticles, respectively. In low Reynolds numbers, the viscous forces are much larger than inertial forces. Therefore, the governing fluid mechanics for the helical robot in low Reynolds numbers are given by the following Stokes equation [22]:

$$
\begin{aligned}
\mu \nabla^{2} \mathbf{u}-\nabla p & =-\mathbf{g} \phi_{\epsilon}\left(r-r_{0}\right), \\
\nabla \cdot \mathbf{u} & =0
\end{aligned}
$$

where $\mathbf{u}$ is the velocity field vector, $\mu, \mathbf{g}$, and $p$ are the viscosity of the medium, the body force of the helical robot on the fluid (applied at $r_{0}$ ), and the scalar pressure field, respectively. Further, $\phi_{\epsilon}\left(r-r_{0}\right)$ is a radially symmetric cutoff

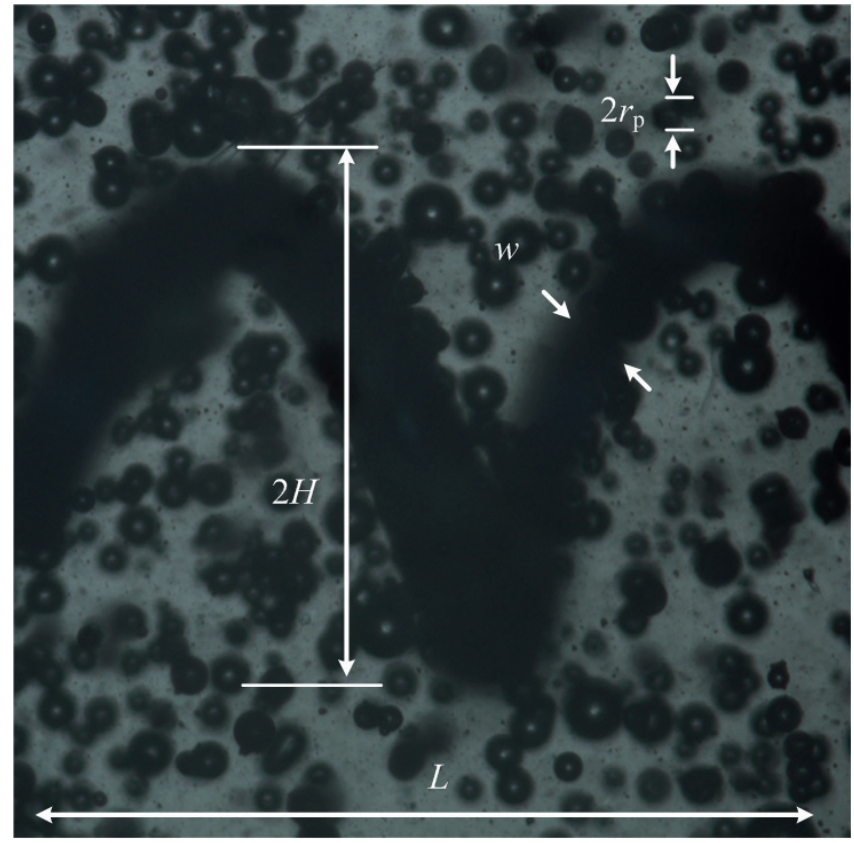

Fig. 2. A microscopic image shows the tail of the helical robot and the surrounding mixture of phosphate buffered saline and 30- $\mu$ m-diameter microparticles (PLA-M, plain, 12-00-304, Micromod Partikeltechnologie $\mathrm{GmbH}$, Rostock-Warnemuende, Germany) with volume concentration $\varphi$. $L$ and $2 H$ indicate the length and diameter of the tail, respectively. $w$ and $r_{\mathrm{p}}$ are the wire diameter of the helix and the radius of the microparticles, respectively. The volume concentration range is $0 \leq \varphi \leq 45 \%$ and the actuation frequency range is $1 \leq f \leq 8 \mathrm{~Hz}$.

function given by

$$
\phi_{\epsilon}\left(r-r_{0}\right)=\frac{15 \epsilon^{4}}{8 \pi\left(r^{2}+\epsilon^{2}\right)^{7 / 2}},
$$

where $\epsilon$ is a parameter that controls the spreading of the cutoff function such that $\int \phi_{\epsilon}(r) \mathrm{d} r=1$. An approximation for the fluid velocity at any point $r_{0}$ is given by

$$
u_{j}\left(r_{0}\right)=\frac{1}{8 \pi \mu} \sum_{n=1}^{N} \sum_{i=1}^{3} S_{i j}^{\epsilon}\left(r_{n}, r_{0}\right) g_{n, i} A_{n},
$$

where $N$ is the number of Stokeslets boundary points located on the surface of the helical robot and the obstacles. Further, $g_{n, i}$ is the $i$ th component of the applied force on the fluid at point $r_{n}$, and $A_{n}$ is a quadratic weight of the $n$th point. In (4), $S_{i j}^{\epsilon}\left(r_{n}, r_{0}\right)$ is the regularized Green's function for Stokes flow and given by

$$
S_{i j}^{\epsilon}\left(r_{n}, r_{0}\right)=\delta_{i j} \frac{r^{2}+2 \epsilon^{2}}{\left(r^{2}+\epsilon^{2}\right)^{3 / 2}}+\frac{\left(r_{i}-r_{0, i}\right)\left(r_{j}-r_{0, j}\right)}{\left(r^{2}+\epsilon^{2}\right)^{3 / 2}},
$$

where $\delta_{i j}$ is the Kronecker delta. Stokeslets boundary points are defined on the surface of the helical robot and the immersed obstacles in the medium. The centerline of the helix is defined as follows:

$$
\begin{aligned}
& x=\left(H-\frac{w}{2}\right) \cos \left(\frac{-2 \pi z_{\mathrm{c}} N}{L}\right), \\
& y=\left(H-\frac{w}{2}\right) \sin \left(\frac{-2 \pi z_{\mathrm{c}} N}{L}\right),
\end{aligned}
$$



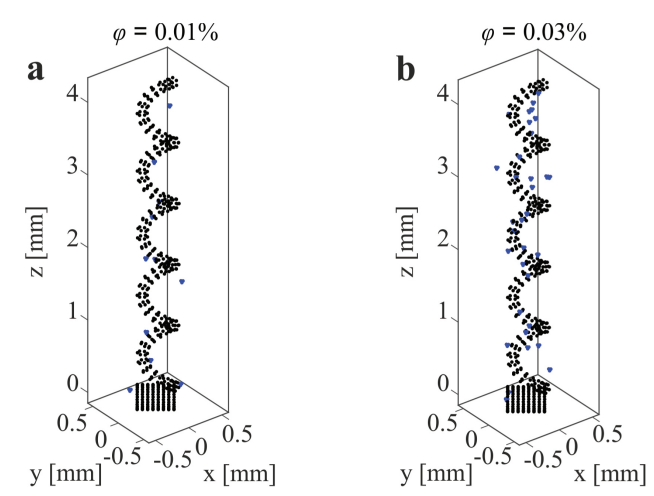

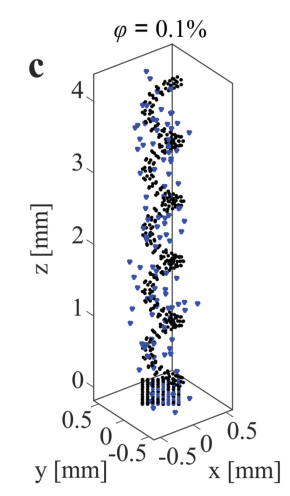

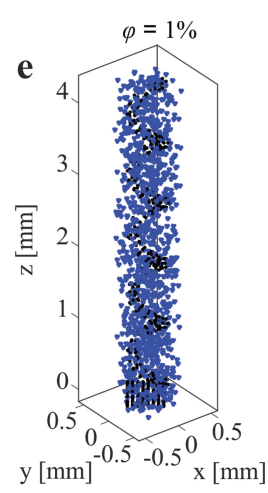

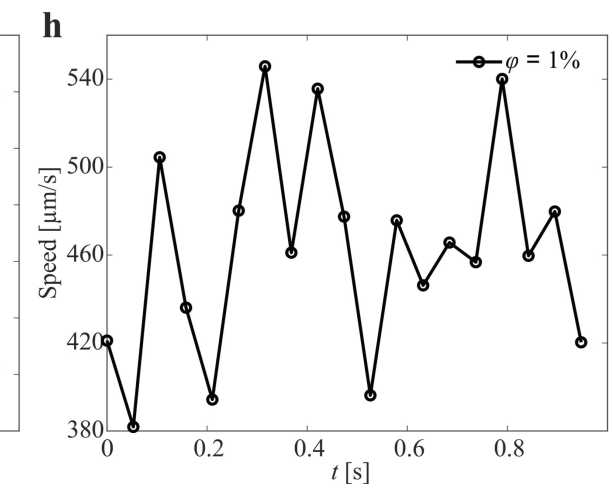

Fig. 3. The swimming speed of the helical robot is calculated for representative volume concentrations of the immersed particles in the medium. The speed is calculated for $\mu=1$ Pa.s, $H=250 \mu \mathrm{m}, L=4 \mathrm{~mm}, w=120 \mu \mathrm{m}, \epsilon=r_{\mathrm{p}} / 4$ and $r_{\mathrm{p}}=15 \mu \mathrm{m}$. The black dots and the blue tetrahedrons represent the helical robot and the immersed obstacles, respectively. (a) $\varphi=0.01 \%$. (b) $\varphi=0.03 \%$. (c) $\varphi=0.1 \%$. (d) $\varphi=0.3 \%$, (e) $\varphi=1 \%$. (f) At $\varphi=0.01 \%$ and $\varphi=0.03 \%$, the average speeds are $52 \mu \mathrm{m} / \mathrm{s}$ and $63 \mu \mathrm{m} / \mathrm{s}$, respectively, for $f=1 \mathrm{~Hz}$. (g) At $\varphi=0.1 \%$ and $\varphi=0.3 \%$, the average speeds are $102 \mu \mathrm{m} / \mathrm{s}$ and $185 \mu \mathrm{m} / \mathrm{s}$, respectively, for $f=1 \mathrm{~Hz}$. (h) At $\varphi=1 \%$ the average speed is $447 \mu \mathrm{m} / \mathrm{s}$, for $f=1 \mathrm{~Hz}$.

where $0 \leq z_{\mathrm{c}} \leq L$, and the distance between the Stokeslets points is $\pi w / N_{\mathrm{cs}}$, where $N_{\mathrm{cs}}$ is the number of points per cross section at radius $w$ from the centerline. The helix is shifted by $\sqrt{r_{\mathrm{c}}^{2}-y(0)^{2}}$ along the $\mathbf{z}$-axis to avoid overlap between the helix and the head. This head also has $2 N_{\mathrm{cs}} r_{\mathrm{c}} / w$ Stokeslets points at each cross-section along the $\mathrm{x}$-axis for $x=\left(-\frac{L_{c}}{2}, \frac{\pi w}{N_{\mathrm{cs}}}, \ldots, \frac{L_{c}}{2}\right), y=z=0$. Finally, the immersed microparticles are approximated by tetrahedrons at random positions $r_{\mathrm{p}, i}$ for $\left(i=1, \ldots, N_{\mathrm{p}}\right)$ and arranged as follows:

$$
\begin{gathered}
\mathbf{r}_{\mathrm{p} i 1}=\mathbf{r}_{\mathrm{p} i} \quad, \quad \mathbf{r}_{\mathrm{p} i 2}=\mathbf{r}_{\mathrm{p} i}+\sqrt{\frac{4}{3}}\left(\begin{array}{l}
0 \\
1 \\
1
\end{array}\right) \\
\mathbf{r}_{\mathrm{p} i 3}=\mathbf{r}_{\mathrm{p} i}+\sqrt{\frac{4}{3}}\left(\begin{array}{l}
1 \\
0 \\
1
\end{array}\right), \mathbf{r}_{\mathrm{p} i 4}=\mathbf{r}_{\mathrm{p} i}+\sqrt{\frac{4}{3}}\left(\begin{array}{l}
1 \\
1 \\
0
\end{array}\right) .
\end{gathered}
$$

The particles are randomly distributed in a volume $V$ with range of $\left(-\frac{R_{x}}{2}, \ldots, \frac{R_{x}}{2}\right),\left(-\frac{R_{y}}{2}, \ldots, \frac{R_{y}}{2}\right)$, and $\left(R_{z 1}, \ldots, R_{z 2}\right)$ along the $\mathbf{x}-, \mathbf{y}^{-}$, and $\mathbf{z}$-axis, respectively, where $R_{x}=2 H+0.1 \mathrm{~mm}, R_{y}=2 H+0.1 \mathrm{~mm}, R_{z 1}=$ $-r_{\mathrm{c}}-0.05 \mathrm{~mm}$ and $R_{z 2}=r_{\mathrm{c}}+L+0.05 \mathrm{~mm}$ are the boundaries of the workspace in which the microparticles are randomly distributed. We assume that the microparticles are stationary and have zero velocities, $u_{i}\left(r_{p i k}\right)=0$ for $i=1, \ldots, N_{\mathrm{p}}$ and $k=1, \ldots, 4$. This assumption is valid when the immersed obstacles are not in close proximity to the helical robot. The velocity of the flow-field caused by the helical robot decreases with the distance from its surface. Therefore, this assumption is valid for immersed obstacles that are not in close proximity to the helical robot.

\section{B. Simulation Results}

The velocity of the helical robot is solved numerically using the inverse of equation (4) to obtain the force at every Stokeslets boundary point. The total force along the propulsion axis of the helical robot is calculated using $F_{z}=$ $\sum_{n=1}^{N} g_{n, 3}$. The velocity of the helical robot is calculated such that $F_{z}=0$. For each concentration, microparticles are randomly distributed and the speed of the helical robot is determined from 20 different simulations. Figs. 3(a)-(e) show representative simulation results of helical propulsion in a viscous heterogenous medium with concentrations of $0.01 \%$, $0.03 \%, 0.1 \%, 0.3 \%$, and $1 \%$, respectively. The speed of the helical robot is obtained by averaging the results over 21 complete cycles. The following simulation parameters are used in our calculations: $\mu=1$ Pa.s, $H=250 \mu \mathrm{m}, L=4$ $\mathrm{mm}, w=120 \mu \mathrm{m}, \epsilon=r_{\mathrm{p}} / 4$ and $r_{\mathrm{p}}=15 \mu \mathrm{m}$.

At $\varphi=0.01 \%$ and $f=1 \mathrm{~Hz}$, the average speed of the helical robot is $52 \mu \mathrm{m} / \mathrm{s}$. A slight increase in the immersed particles to $0.03 \%$ results in an average speed of $63 \mu \mathrm{m} / \mathrm{s}$ at $f=1 \mathrm{~Hz}$, as shown in Fig. 3(f). At $\varphi=0.1 \%$ and $\varphi=$ 


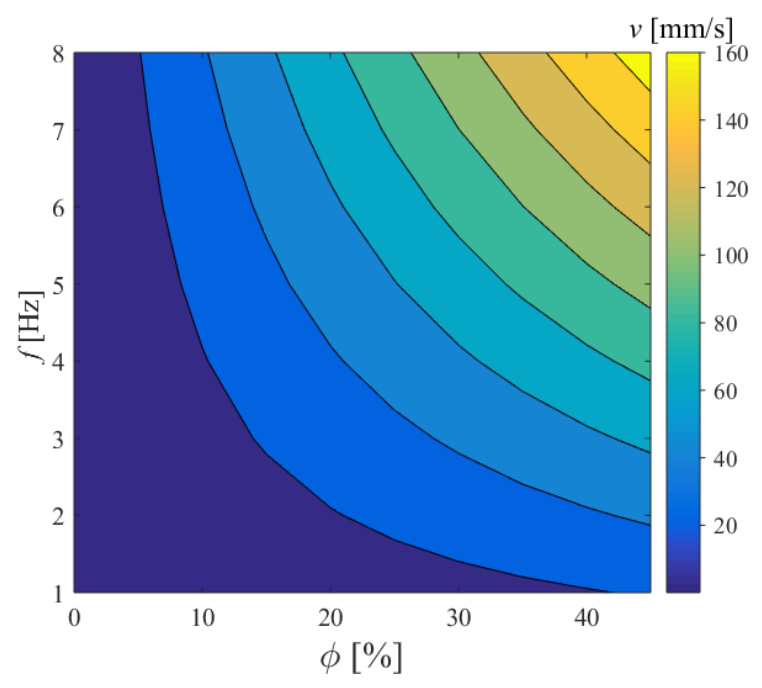

Fig. 4. The numerical model predicts propulsion enhancement with the concentration of the immersed obstacles and the actuation frequency of the external magnetic field. The average swimming speed of a helical robot is calculated for volume concentration and actuation frequency ranges of $0 \leq \varphi \leq 45 \%$ and $1 \leq f \leq 8 \mathrm{~Hz}$, respectively. At actuation frequencies of $1 \mathrm{~Hz}$ and $8 \mathrm{~Hz}$, the swimming speed increases by factors of 2 and 1.4 for each $5 \%$ increase in the concentration of the obstacles. The average speeds are calculated for $\mu=1$ Pa.s, $H=250 \mu \mathrm{m}, L=4 \mathrm{~mm}, w=120 \mu \mathrm{m}$, $\epsilon=r_{\mathrm{p}} / 4$ and $r_{\mathrm{p}}=15 \mu \mathrm{m}$.

$0.3 \%$, the average speeds of the helical robot are $102 \mu \mathrm{m} / \mathrm{s}$ $185 \mu \mathrm{m} / \mathrm{s}$, respectively, at $f=1 \mathrm{~Hz}$ (Fig. 3(g)). The average speed of the helical robot increases to $447 \mu \mathrm{m} / \mathrm{s}$ for $\varphi=1 \%$ and under the influence of rotating magnetic field at $1 \mathrm{~Hz}$, as shown in Fig. 3(h). Fig. 4 shows the influence of the volume concentration of the obstacles and the actuation frequency on the swimming speed of the helical robot for $0 \leq \varphi \leq 45 \%$ and $1 \leq f \leq 8 \mathrm{~Hz}$. This simulation result predicts that the swimming speed linearly increase not only with the actuation frequency but also with the concentration of the immersed obstacle in the medium. At $f=1 \mathrm{~Hz}$, the swimming speed is increased by a factor of 2 for an increase of $5 \%$ in the concentration of the obstacles. At $f=8 \mathrm{~Hz}$, the swimming speed is also increased by a factor of 1.4 for a $5 \%$ increase in the concentration. The speed of the helical robot is measured experimentally to validate the results of the numerical model.

\section{EXPERIMENTAL RESULTS}

The theoretical prediction of the regularized Stokeslets model are compared to experimental results at representative volume concentrations. A permanent magnet-based robotic system is used to actuate the helical robot [23].

\section{A. System Description}

4-mm-long helical robots with outer-diameter of $500 \mu \mathrm{m}$ are contained inside a polyvinyl chloride catheter segment with inner-diameter of $4 \mathrm{~mm}$. The helical robot consists of a helical body attached to a cylindrical permanent magnet with magnetization perpendicular to the helix axis. The catheter is filled with a mixture of phosphate buffered saline (PBS) and polystyrene microparticles (PLA-M, plain, 12-00-304, Micromod Partikeltechnologie GmbH, Rostock-Warnemuende,

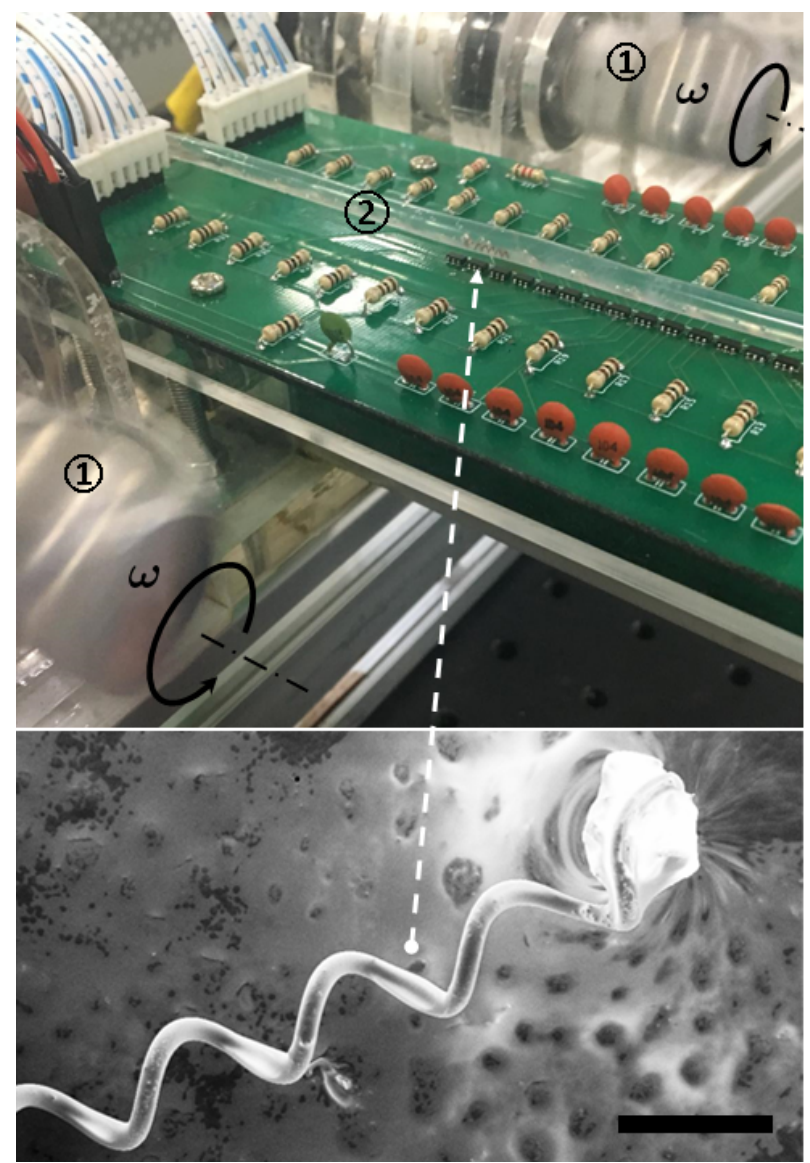

Fig. 5. A permanent magnet-based robotic system is used to characterize the influence of the immersed obstacles on the helical propulsion. The system consists of two rotating dipole fields (1) and the helical robot is contained inside a catheter segment(2) between the permanent magnets [23]. The scale bar is $500 \mu \mathrm{m}$.

Germany) with average diameter of $30 \mu \mathrm{m}$. The density and viscosity of the PBS are $995 \mathrm{~kg} . \mathrm{m}^{-3}$ and 0.888 Pa.s. Therefore, Reynolds number is calculated as, $R e=\rho v L / \mu \simeq$ 0.044 (average speed is $10 \mathrm{~mm} / \mathrm{s}$ for actuation frequency of $1 \mathrm{~Hz}$ ), where $\rho$ and $v$ are the density of the medium and the speed of the helical robot, respectively. The catheter is fixed between two rotating permanent $\mathrm{NdFeB}$ magnets, at a distance of $15 \mathrm{~mm}$ (Fig. 5) and magnetic field of $5.5 \mathrm{mT}$ is measured at the position of the helical robot. The magnets have axial magnetization and their diameter and length are $20 \mathrm{~mm}$ and $20 \mathrm{~mm}$, respectively. Rotations of these magnets are synchronized using DC motors (2322 980, Maxon Motor, Sachseln). This synchronization decreases the pulling force along the lateral directions of the helical robot. Motion of the helical robot is tracked with a high-speed camera (avA100120kc, Basler Area Scan Camera, Basler AG, Ahrensburg, Germany) and a feature tracking algorithm to characterize its swimming speed.

\section{B. Frequency Response Characterization}

The concentration range of the microparticles in the mixture is $5 \%$ to $45 \%$. The experimental results show that helical propulsion is enhanced as the concentration of the 


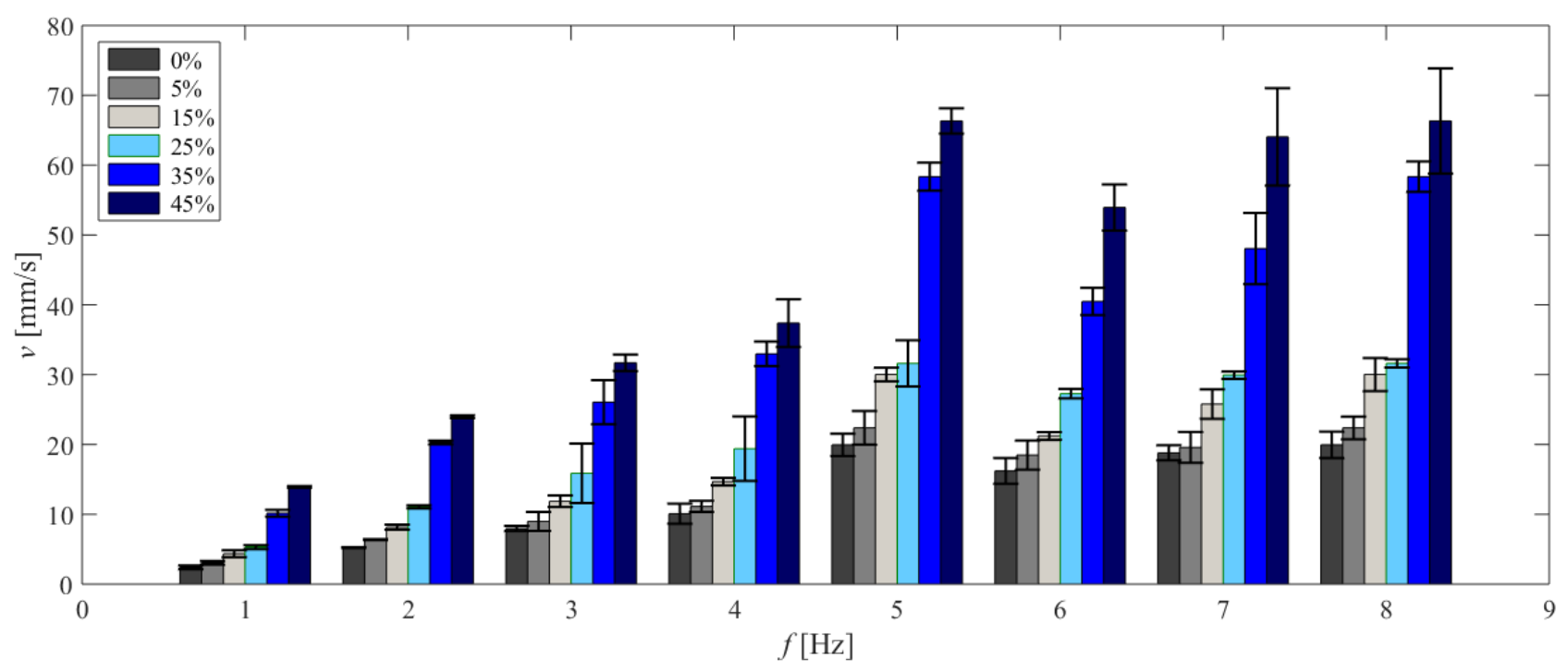

Fig. 6. The influence of the volume concentration $(\varphi)$ of the immersed obstacles and the actuation frequency $(f)$ on the swimming speed of helical robots is characterized experimentally. Robots are controlled to swim between the rotating dipole fields inside a mixture of phosphate buffered saline and polystyrene microparticles. (a) The swimming speed of the helical robot increases with the actuation frequency. (b) The swimming speed of the helical robot increases with the concentration of the immersed obstacles in the medium. The average speeds and standard deviations are calculated from 3 trials at each frequency and for each concentration.

microparticles increases in the medium. Average speeds and standard deviations are calculated from three trial at each actuation frequency and for each concentration. For $\varphi=0 \%$ and $f=1 \mathrm{~Hz}$, the average swimming speed is measured as $2.4 \pm 0.34 \mathrm{~mm} / \mathrm{s}$ and increases to $13.9 \pm 0.15$ $\mathrm{mm} / \mathrm{s}$ for $\varphi=45 \%$, as shown in Figs. 6(a) and 6(b). The propulsion enhancement is also preserved with the increase in the actuation frequency. For $\varphi=0 \%$ and $f=2 \mathrm{~Hz}$, the average swimming speed is measured as $5.2 \pm 0.07 \mathrm{~mm} / \mathrm{s}$ and increases to $23.9 \pm 2.6 \mathrm{~mm} / \mathrm{s}$ for $\varphi=45 \%$. Similarly, for $\varphi=0 \%$ and $f=3 \mathrm{~Hz}$, the average swimming speed is measured as $7.9 \pm 0.47 \mathrm{~mm} / \mathrm{s}$ and increases to $31.7 \pm 1.48$ $\mathrm{mm} / \mathrm{s}$ for $\varphi=45 \%$.

Our measurements and simulation results are in qualitative agreement and show propulsion enhancement with the concentration of the obstacles. At actuation frequency of 1 $\mathrm{Hz}$, the numerical model and experimental results show that the swimming speed of the helical robot is $2.42 \mathrm{~mm} / \mathrm{s}$ and $3.04 \pm 0.34 \mathrm{~mm} / \mathrm{s}$, respectively, at $\varphi=5 \%$. At $\varphi=15 \%$, the calculated and measured speeds of the helical robot are 4.8 $\mathrm{mm} / \mathrm{s}$ and $4.3 \pm 0.66 \mathrm{~mm} / \mathrm{s}$, respectively. At $\varphi=25 \%$, we observe a relatively large deviation between simulation and experimental results. The calculated and measured speeds of the helical robot are $11.8 \mathrm{~mm} / \mathrm{s}$ and $5.3 \pm 0.35 \mathrm{~mm} / \mathrm{s}$, respectively. At $\varphi=35 \%$, the calculated and measured speeds of the helical robot are $16.6 \mathrm{~mm} / \mathrm{s}$ and $10.1 \pm 0.66 \mathrm{~mm} / \mathrm{s}$, respectively. At $\varphi=45 \%$, the calculated and measured speeds of the helical robot are $21.3 \mathrm{~mm} / \mathrm{s}$ and $13.9 \pm 0.17 \mathrm{~mm} / \mathrm{s}$, respectively. At actuation frequency of $4 \mathrm{~Hz}$, the calculated and measured speeds of the helical robot are $9.68 \mathrm{~mm} / \mathrm{s}$ and $11.1 \pm 1.1 \mathrm{~mm} / \mathrm{s}$ for $\varphi=5 \%$ and $28.6 \mathrm{~mm} / \mathrm{s}$ and $14.6 \pm 0.7$ $\mathrm{mm} / \mathrm{s}$ for $\varphi=15 \%$, respectively. we also obverse deviations between measurements and theoretical predictions at other concentrations. For $\varphi=25 \%$, the calculated and measured speeds are $47.5 \mathrm{~mm} / \mathrm{s}$ and $19.4 \pm 5.8 \mathrm{~mm} / \mathrm{s}$, respectively. Similarly for $\varphi=35 \%$, the calculated and measured speeds are $66.5 \mathrm{~mm} / \mathrm{s}$ and $32.9 \pm 2.2 \mathrm{~mm} / \mathrm{s}$, respectively. Finally for $\varphi=45 \%$, the calculated and measured speeds are $85.4 \mathrm{~mm} / \mathrm{s}$ and $44.0 \pm 2.3 \mathrm{~mm} / \mathrm{s}$, respectively. Therefore, the calculated speed of the helical robot is greater than the measured speed by approximately a factor of 2 . At actuation frequency of $8 \mathrm{~Hz}$, the calculated speeds are also greater than the measured speeds except for one concentration. For $\varphi=5 \%$, the calculated and measured speeds are $19.35 \mathrm{~mm} / \mathrm{s}$ and $22.3 \pm 2.1 \mathrm{~mm} / \mathrm{s}$, respectively.

We observe a deviation with a factor of approximately 2.4 between simulation and experimental results for $\varphi>5 \%$. We attribute the difference between experimental results and theoretical predictions to the error in the parameters entered to the numerical models. In addition, we assume that the immersed microparticles are fixed and have zero initial velocity. Fixed obstacles enhance the propulsion compared to free obstacles, as the helical robot pushes itself and move forward by the fixed obstacles. In experiments, the microparticles are free to move based on the flow-field caused by the helical robot. This velocity flow-field influences the surrounding obstacles based on their distance to the surface of the helical robot. Therefore, our assumption is only valid for microparticles that are not in close proximity to the helical robot and the non-zero velocity of microparticles near the helical robot result deviation between experiments and theoretical prediction. The deviation between the experimental and theoretical results are more evident at relatively high actuation frequency. At actuation frequencies of $1 \mathrm{~Hz}, 4$ 
$\mathrm{Hz}$, and $8 \mathrm{~Hz}$, the calculated speeds are greater than the measurements by factors of $1.4,2$, and 2.4 , respectively. This frequency dependence indicates that the velocity of the immersed obstacles have to be incorporated into the model, especially at high actuation frequency. The velocity flow-field of the helical robot depends on its actuation frequency, and at low actuation frequency the microparticles are influenced by a relatively low flow-field. Therefore, our model provides qualitative agreement with the experiments at low actuation frequency.

\section{CONCLUSions ANd Future Work}

Helical propulsion in a heterogenous viscous medium is enhanced by increasing the concentration of the immersed obstacles. Our simulation and experimental results show that the swimming speed of the helical robot increases linearly with the concentration of the immersed obstacles and actuation frequency. Simulations are implemented on a numerical model based on the regularised Sokeslets method to predict the Stokes flow at boundary points on the helical robot and the immersed obstacles. Our numerical model predicts that the swimming speed is enhanced by a factor of 2 for a $5 \%$ increase in the concentration of the immersed obstacles, at rotation frequency of $1 \mathrm{~Hz}$, whereas experimental results show enhancement in the speed by a factor of 1.48. At relatively high actuation frequency $f=8 \mathrm{~Hz}$, the numerical model and experimental result show propulsion enhancement by factors of 1.4 and 1.3 in the swimming speed, respectively.

As part of our future work, we will modify our numerical model to include the influence of free and fixed obstacles in the medium. This modification is essential to investigate the deviations between the measurements and theoretical predictions of this study. We will also investigate the influence of the size of the immersed obstacles on the helical propulsion theoretically and experimentally. The size of the immersed obstacles is likely to vary in several in vivo conditions. We will also control helical robots against flowing streams of heterogenous viscous fluids and characterize the effect of time-varying flow rates on their propulsion. In addition, our permanent magnet-based robotic system will be used in the localization and motion control of helical robots. This modification will be achieved by adding arrays of Hall-effect sensors to localize the helical robots based on its field and the pre-calculated field of the actuating magnets. We will also achieve closed-loop motion control of helical robots inside ex-vivo models of blood vessels.

\section{ACKNOWLEDGMENT}

The authors would like to thank Mr. Y. Hamed for assistance with the experimental work and the preparation of Fig. 1(b).

\section{REFERENCES}

[1] A. Ghosh and P. Fischer, "Controlled propulsion of artificial magnetic nanostructured propellers," Nano Letters, vol. 9, pp. 2243-2245, June 2009.

[2] J. J. Abbott, K. E. Peyer, L. Dong, and B. Nelson, "How should microrobots swim?" The International Journal of Robotics Research, vol. 28, no. 11-12, pp. 1434-1447, 2009.
[3] E. M. Purcell, "Life at low Reynolds number," American Journal of Physics, vol. 45, no. 1, pp. 3-11, 1977.

[4] M. P. Kummer, J. J. Abbott, B. E. Kartochvil, R. Borer, A. Sengul, and B. J. Nelson, "OctoMag: an electromagnetic system for 5-DOF wireless micromanipulation," IEEE Transactions on Robotics, vol. 26, no. 6, pp. 1006-1017, December 2010

[5] B. J. Nelson, I. K. Kaliakatsos, and J. J. Abbott, "Microrobots for minimally invasive medicine," Annual Review of Biomedical Engineering, vol. 12, pp. 55-85, April 2010.

[6] T. W. R. Fountain, P. V. Kailat, and J. J. Abbott, "Wireless control of magnetic helical microrobots using a rotating-permanent-magnet manipulator," in Proceedings of the IEEE International Conference on Robotics and Automation (ICRA), pp. 576-581, Alaska, USA, May 2010.

[7] E. Diller and M. Sitti, "Three-dimensional programmable assembly by untethered magnetic robotic micro-grippers," Advanced Functional Material, vol. 24, no. 28, pp. 4397-4404, July 2014.

[8] A. W. Mahoney, D. L. Cowan, K. M. Miller, and J. J. Abbott, "Control of untethered magnetically actuated tools using a rotating permanent magnet in any position," in Proceedings of the IEEE International Conference on Robotics and Automation (ICRA), pp. 3375-3380, Minnesota, USA, May 2012.

[9] K. E. Peyer, L. Zhang, and B. J. Nelson, "Bio-inspired magnetic swimming microrobots for biomedical applications," Nanoscale, vol. 5, no. 4, pp. 1259-1272, November 2012.

[10] W. F. Paxton, K. C. Kistler, C. C. Olmeda, A. Sen, S. K. St. Angelo, Y. Cao, T. E. Mallouk, P. E. Lammert, and V. H. Crespi, "Catalytic nanomotors: autonomous movement of striped nanorods," Journal of the American Chemical Society, vol. 126, no. 41, pp. 13424-13431, September 2004.

[11] W. Wang, L. A. Castro, M. Hoyos, and T. E. Mallouk, "Autonomous motion of metallic microrods propelled by ultrasound," ACS Nano, vol. 6, no. 7, pp. 6122-6132, May 2012,

[12] P. Calvo-Marzal, S. Sattayasamitsathit, S. Balasubramanian, J. R. Windmiller, C. Dao, and J. Wang, "Propulsion of nanowire diodes," Chemical Communications, vol. 46, no. 10, pp. 1623-1624, February 2010.

[13] M. Pumera, "Electrochemically powered self-propelled electrophoretic nanosubmarines," Nanoscale, vol. 2, no. 9, pp. 1643-1649, May 2010.

[14] I. S. M. Khalil, V. Magdanz, S. Sanchez, O. G. Schmidt, and S. Misra, "The control of self-propelled microjets inside a microchannel with time-varying flow rates," IEEE Transactions on Robotics, vol. 30, no. 1, pp. 49-58, February 2013.

[15] A. M. Leshansky, "Enhanced low-reynolds-number propulsion in heterogeneous viscous environments," Physical Review E, vol. 80, 051911, November 2009.

[16] E. Lauga, W. R. DiLuzio, G. M. Whitesides, and H. A. Stone, "Swimming in circles: motion of bacteria near solid boundaries," Biophysical Journal, vol. 90, pp. 400-412, January 2006.

[17] N. D. Nelson, J. Delacenserie, and J. J. Abbott, "An empirical study of the role of magnetic, geometric, and tissue properties on the turning radius of magnetically driven screws," in Proceedings of the IEEE International Conference on Robotics and Automation (ICRA), pp. 5372-5377, Karlsruhe, Germany, May 2013.

[18] F. Ullrich, F. Qiu, J. Pokki, T. Huang, S. Pane, and B. J. Nelson, "Swimming characteristics of helical microrobots in fibrous environments," in Proceedings of the IEEE RAS/EMBS International Conference on Biomedical Robotics and Biomechatronics (BioRob), pp. 470-475, UTown, Singapore, June 2016.

[19] H. C. Berg and L. Turner, "Movement of microorganisms in viscous environments," Nature, vol. 278, pp. 349-351, March 1979.

[20] I. S. M. Khalil, V. Magdanz, Y. Hamed, M. Toubar, A. Klingner and S. Misra, "Characterization of flagellar propulsion of soft microrobotic sperm in a viscous heterogeneous medium," Frontiers in Robotics and $A I$, vol. 6, no. 65, July 2019.

[21] R. Cortez, "The method of regularized Stokeslets," SIAM Journal on Scientific Computing, vol. 23, no. 4, pp. 1204-1225, July 2001.

[22] R. Cortez, L. Fauci, and A. Medovikov, "The method of regularized Stokeslets in three dimensions: Analysis, validation, and application to helical swimming," Physics of Fluids, vol. 17, no. 031504, February 2005 .

[23] I. S. M. Khalil, A. F. Tabak, K. Sadek, D. Mahdy, N. Hamdi, and M. Sitti, "Rubbing against blood clots using helical robots: modeling and in vitro experimental validation," IEEE Robotics \& Automation Letters, vol. 2, no. 2, pp. 927-934, April 2017. 